\title{
Critical Appraisal of the MTr Assay in the Presence of Rottlerin and Uncouplers
}

\section{Emanuela Maioli, Claudia Torricelli, Vittoria Fortino, Filippo Carlucci, Valentina Tommassini, and Adriana Pacini}

\begin{abstract}
Rottlerin is a natural product isolated from Mallotus philippinensis. This polyphenolic compound, originally described as a selective inhibitor of PKC $\delta$, can inhibit many other PKC-unrelated kinases and has a number of biological actions, including mitochondrial uncoupling effects. We recently found that Rottlerin inhibits the transcription factor nuclear factor $\kappa \mathrm{B}$ in different cell types, causing downregulation of cyclin Dl and growth arrest. The present study was carried out to clarify the surprising lack of effect of Rottlerin on MCF-7 cell viability, assessed by the 3-(4,5-dimethylthiazol-2-yl)-2,5-diphenyl tetrazolium bromide (MTT) test. We found that Rottlerin causes overestimation of the MTT test, leading to inconsistent results between cell number and cell viability. Rottlerin, however, strongly differs from other antioxidant polyphenols, which directly reduce tetrazolium salts, since it does not exhibit any reactivity toward the tetrazolium salts in vitro nor does it modulate lactate dehydrogenase activity. The interference in the MTT assay occurred only in cultured cells, concomitantly with a decrease in the energy charge. Because the same MTT overestimation was observed in the presence of uncoupling agents, we conclude that the Rottlerin artifact is linked to its uncoupling action that, by accelerating oxidative chain, accidentally results in enhanced MTT reduction. These results suggest caution in the use of the MTT assay in the presence of Rottlerin and uncouplers in general.
\end{abstract}

Keywords: Rottlerin, MCF-7 cell, MTT, LDH, FCCP, mitochondrial uncoupling.

\section{Introduction}

Rottlerin (also called mallotoxin or kamala), is a 5,7-dihydroxy2,2-dimethyl-6-(2,4,6-trihydroxy-3-methyl-5-acetylbenzyl)-8cinnamoyl-1,2-chromene, a pigmented plant product isolated from Mallotus philippinensis (Fig. 1). Since 1994, Rottlerin has

Shulin Li (ed.), Biological Procedures Online, Volume 11, Number 1

(C) to the author(s) 2009

DOI: 10.1007/s12575-009-9020-1 URL: springerprotocols.com; springerlink.com 
<smiles>CC(=O)c1c(O)c(C)c(O)c(Cc2c(O)c3c(c(C(=O)/C=C/c4ccccc4)c2O)OC(C)(C)C=C3)c1O</smiles>

Fig. 1. Rottlerin structure.

been used as a PKC $\delta$ inhibitor (1) although the selectivity of Rottlerin in inhibiting the PKC $\delta$ isoform has been recently questioned $(2,3)$ and ascribed to a likely indirect effect mediated by mitochondrial uncoupling and decrease in ATP content (4). Our laboratory showed for the first time that Rottlerin can prevent, independently from $\mathrm{PKC}$, the activation of the transcription factor nuclear factor $\kappa \mathrm{B}(\mathrm{NF \kappa B})$, induced by either phorbol ester or oxidative stress in MCF-7 cells (5), HaCaT keratinocytes (6) and HT-29 cells (unpublished results), whose growth resulted to be arrested because of downregulation of cyclin Dl, at both the protein and mRNA levels. Although the molecular mechanism is not definitively clarified, the prevention of the NFKB activation process was likely achieved through both Rottlerin inhibition of protein kinases $(7,8)$ and Rottlerin free radicals scavenging activity (9). Indeed, NFkB can be activated by a number of pathways and is a redox-sensitive transcription factor for key molecules involved in inflammation, cancer progression, cell cycle control, and protection against apoptosis (10).

However, in our previous paper (5), we found that MCF-7 cell viability was not altered by a 24 -h Rottlerin treatment, a result that was in evident conflict with the inhibition of NFkB and cell proliferation, as evaluated by $\left[{ }^{3} \mathrm{H}\right]$-thymidine incorporation into DNA. Since the measurement of cell viability was based on the reduction of 3-(4,5-dimethylthiazol-2-yl)-2,5-diphenyl tetrazolium bromide (MTT) to formazan crystal by mitochondrial dehydrogenases (11), in the current study we revisited our previous results, checking for a possible interference of Rottlerin in the MTT assay.

\section{Materials and methods}

Materials: All chemicals and materials for cell culture (unless otherwise indicated) were obtained from Sigma (Milan, Italy). Lactate dehydroge- 
nase (LDH) assay kit was purchased from Sclavo Diagnostics (Siena, Italy). Rottlerin was dissolved in dimethyl sulfoxide (DMSO). Carbonylcyanide-4-(trifluoromethoxy)-phenylhydrazone (FCCP) was dissolved in $95 \%$ ethanol.

Cells and culture conditions: MCF-7 cells, purchased by Istituto Zooprofilattico Sperimentale della Lombardia e dell'Emilia-Romagna, Brescia, Italy, were grown in a humidified atmosphere $(95 \%$ air $/ 5 \%$ $\left.\mathrm{CO}_{2}\right)$ at $37^{\circ} \mathrm{C}$ in MEM containing $10 \% \mathrm{FBS}$, Na-pyruvate $(1 \mathrm{mM})$, and antibiotics.

Primary human microvascular endothelial cells (HMVEC), purchased by LONZA (Milano, Italy), were cultured in EBM-2 medium supplemented with EGM-2 Single Quots (LONZA), and experiments were performed on cultures from passages 3 to 9 .

After reaching subconfluence, cells were incubated in serumfree medium for $24 \mathrm{~h}$ and then subjected to treatments in $2.5 \%$ serum.

Cell counting: The number of cells, cultured in $25-\mathrm{cm}^{2}$ culture flasks (Falcon, Perugia, Italy) was evaluated by detaching with trypsin solution $(0.05 \%$ trypsin- $0.02 \%$ sodium EDTA) and counting using a Bürker chamber and trypan blue solution $(0.2 \% w / v$ final dye concentration). Viable MCF-7 cell number counts were obtained at 1-, 2-, 4-, 15-, and 24-h incubation in the presence or absence of Rottlerin 5 and $20 \mu \mathrm{M}$.

Viable HMVEC cell number counts were obtained at 24-h incubation in the presence or absence of $20 \mu \mathrm{M}$ Rottlerin.

Three replicate counts were determined by the same operator at each time point. The data were presented as proportional viability (\%) by comparing the Rottlerin-treated with the vehicletreated cells, whose viability was assumed to be $100 \%$.

MTT assay: Cell viability was assessed using the MTT colorimetric assay. MTT is taken up into cells by endocytosis or protein-facilitated mechanism and reduced, mainly by mitochondrial enzymes, to yield a purple formazan product which is largely impermeable to cell membranes, thus resulting in its accumulation within living cells. Solubilization of the cells results in the liberation of the purple product which can be detected using a colorimetric measurement. The ability of cells to reduce MTT provides an indication of the mitochondrial integrity and activity which, in turn, may be interpreted as a measure of cell number/proliferation/viability/survival/toxicity.

Operatively, $100 \mu \mathrm{l}$ of cell suspension was inoculated to each well of 96-well plates at the density of $2 \times 10^{4}$ cells /well (the area of each well was $0.32 \mathrm{~cm}^{2}$ ). After $24 \mathrm{~h}$ of culture, the medium was removed by aspiration and replaced with $100 \mu$ of experimental medium. The treatments were performed with 5 and $20 \mu \mathrm{M}$ Rottlerin for $1,2,4,15$, and $24 \mathrm{~h}$. After incubation, cells were observed under a contrast phase microscope before adding MTT solution, prepared fresh as $5 \mathrm{mg} / \mathrm{ml}$ in $\mathrm{H}_{2} \mathrm{O}$, filtered through a $0.22-\mu \mathrm{m}$ filter, and kept for $5 \mathrm{~min}$ at $37^{\circ} \mathrm{C}$. MTT solution 
$(10 \mu \mathrm{l})$ was added to each well, and the plates were incubated in the dark for $4 \mathrm{~h}$ at $37^{\circ} \mathrm{C}$.

To check for the direct effect of Rottlerin on the formazan production, a parallel set of experiments was carried out in cellfree plates. The followed procedure was the same as described above with some modifications: (a) The Rottlerin doses were 5, 20,50 , and $100 \mu \mathrm{M}$; (b) the incubation time with MTT was prolonged from 4 up to $24 \mathrm{~h}$; (c) different media (DMEM, MEM, and RPMI 1640), enriched or not with $10 \%$ serum or $20 \mu \mathrm{M}$ $\mathrm{NAD}$ or $\mathrm{NADH}$, were tested.

In another set of experiments MCF-7 cells were treated for 30 min with equal doses ( 5 and $20 \mu \mathrm{M}$ ) of Rottlerin or the chemical uncoupler FCCP, before incubation with $10 \mu$ of MTT for $\mathrm{l}$ h at $37^{\circ} \mathrm{C}$.

HMVEC were treated for $24 \mathrm{~h}$ with $20 \mu \mathrm{M}$ Rottlerin before incubation with MTT for $4 \mathrm{~h}$.

At the end of each experiment, the medium was removed by inverting and tapping the plates and $100 \mu \mathrm{l}$ solution of $4 \% \mathrm{HCl}$ $\mathrm{l} \mathrm{N}$ in isopropanol was added to immediately dissolve the formazan crystals. Absorbance at $570 \mathrm{~nm}$ was read on a Multiwell scanning spectrophotometer (Sclavo, Siena, Italy), and the results were expressed as a percentage (\%) of the control (vehicle alone).

The medium from the plates without cells was collected and both read directly and centrifuged before solubilization of (eventual invisible) formazan crystals. In this experiment, the reducing agent dithiothreitol was used as a positive control.

$L D H$ assay: $\mathrm{LDH}$ assay was performed in culture medium of untreated confluent cells by using a commercial kit (Sclavo Diagnostics, Siena) based on the transformation of pyruvate to lactate by $\mathrm{LDH}$, at $\mathrm{pH}$ 7.5 , in the presence of NADH coenzyme. The transformation of $\mathrm{NADH}$ to NAD+ is accompanied by a decrease in absorbance (A) at $340 \mathrm{~nm}$, which correlates with the $\mathrm{LDH}$ activity. The change of absorbance, in the absence or presence of different doses of Rottlerin, was recorded over a 0.5 - to $4.5-\mathrm{min}$ period, and the relative $\Delta A / \mathrm{min}$ was calculated. The change in absorbance was converted to LDH international units per liter $(\mathrm{U} / \mathrm{l})$ by the following calculations: $\Delta A / \mathrm{min}$. ( $\mathrm{tV} \cdot 1,000 / \mathrm{EMC} \cdot l \cdot \mathrm{sV})$, where $\mathrm{tV}$ is the total volume, EMC is the NADH extinction micromolar coefficient $\left(6.22 \mathrm{~cm}^{2} \mu \mathrm{mol}\right.$ at $340 \mathrm{~nm}), l$ is the light pathlength $(1 \mathrm{~cm})$, and $\mathrm{sV}$ is the sample volume.

Evaluation of nucleotide content: Nucleotides were evaluated as previously described (12). The cells $\left(5 \times 10^{6}\right)$ were homogenized in $2.7 \mathrm{~N}$ perchloric acid in $0.5-\mathrm{ml}$ tubes using a Sigma nylon motor pestle. Extracts were then centrifuged $(12,000 \times g$ for $10 \mathrm{~min})$ in a cooled microfuge. The supernatant was neutralized with $2.7 \mathrm{NKOH}$; potassium perchlorate was removed by a subsequent centrifugation at $12,000 \times g$ for $3 \mathrm{~min}$. Aliquots of the extracts were analyzed by capillary zone electrophoresis (CZE), as afterward reported. The method permits to determine high-energy phosphates (ATP, ADP, and AMP) from which it is possible to calculate the energy charge of adenylates. 
Capillary zone electrophoresis: For electrophoretic separations a Beckman Coulter P/ACE MDQ instrument (Beckman Coulter, Fullerton, CA, USA) was used. Analysis were performed in a Beckman Coulter eCAP ${ }^{\mathrm{TM}}$ uncoated fused-silica capillary $(65.0 \mathrm{~cm} \times$ $75 \mu \mathrm{m}$ i.d.), with the window at a distance of $55.0 \mathrm{~cm}$. The results were read over the range 190-300 $\mathrm{nm}$ and analyzed at $254 \mathrm{~nm}$. Between runs capillary was washed with $0.1 \mathrm{~mol} / \mathrm{l} \mathrm{NaOH}$ for $30 \mathrm{~s}$ and running buffer for $60 \mathrm{~s}$. The background electrolyte was borate buffer $(20 \mathrm{mM})$, containing SDS $(30 \mathrm{mM})$. The conditions were $\mathrm{pH}$ $10.00,20 \mathrm{kV}$, and 1.0 psi for $5 \mathrm{~s}$ of pressure injection at $25^{\circ} \mathrm{C}$, for sample and standard solutions. The electric field was $306 \mathrm{~V} /$ $\mathrm{cm}$ with a current of approximately $120 \mu \mathrm{A}$.

Statistical analysis: The significant difference between control and treated cells was statistically analyzed by paired Student's $t$ test $(p<0.05)$.

\section{Results}

\subsection{Rottlerin interference in the MTT assay}

Consistently with the decrease in $\left[{ }^{3} \mathrm{H}\right]$-thymidine incorporation into DNA previously observed (5), 5 and $20 \mu \mathrm{M}$ Rottlerin caused a time and a dose-dependent decrease in cell number, although the dose of $5 \mu \mathrm{M}$ did not cause statistically significant changes before $24 \mathrm{~h}$ (Fig. 2a). However, as shown in Fig. 2b, the MTT colorimetric assay strongly underestimated the growth inhibitory activity of $20 \mu \mathrm{M}$ Rottlerin and absolutely reversed the result, suggesting a direct Rottlerin action on the tetrazolium salt. Rottlerin $(5 \mu \mathrm{M})$ exhibited the same trend of $20 \mu \mathrm{M}$ although the results were not statistically significant at any time point. When the intensity of the reduced product color at $570 \mathrm{~nm}$ was normalized to cell number (absorbance/cell number), the stimulation of MTT reduction by Rottlerin was evident (Table 1 ). Rottlerin, however, did not reduce MTT in vitro at doses up to $100 \mu \mathrm{M}$ and for prolonged times $(24 \mathrm{~h})$, in several incubation conditions, i.e., in different culture media and in the presence or absence of serum, $\mathrm{NAD}$ or NADH (not shown).

As shown in Fig. 3, an overestimation of the MTT assay with respect to cell number was also observed in HMVEC, after a 24-h treatment with $20 \mu \mathrm{M}$ Rottlerin.

As shown in Table 2, the kinetic of NADH oxidation by LDH is unchanged in the presence of 5 (not shown) and $20 \mu \mathrm{M}$ Rottlerin with respect to the control, as it results from the very similar $\Delta A$ at the different times, although the absolute optical densities (OD; 

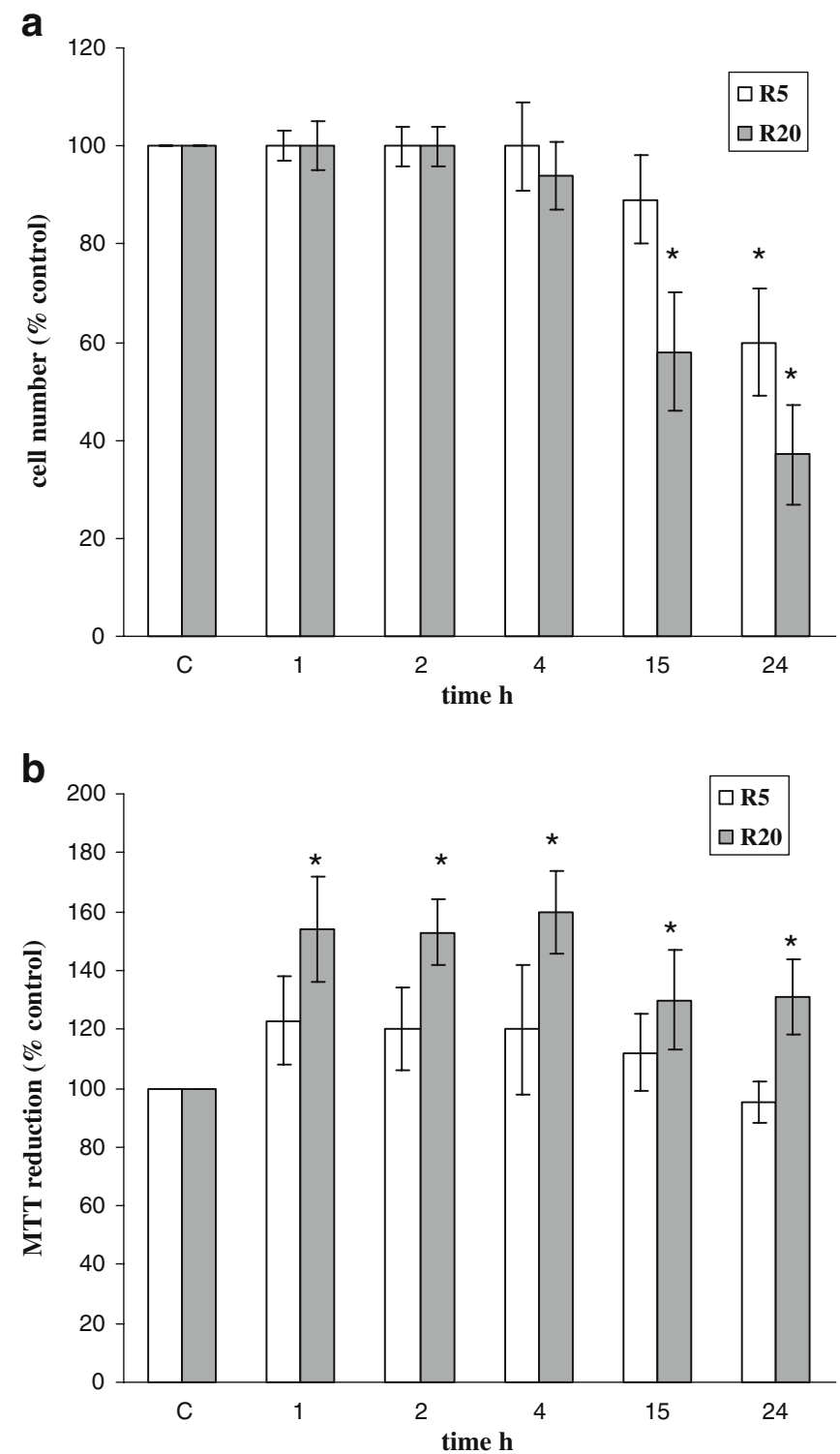

Fig. 2. Effect of Rottlerin on cell number and viability assessed by direct cell counting and MTT assay, respectively. MCF-7 cells were treated with 5 and $20 \mu \mathrm{M}$ Rottlerin ( $R 5$ and $R 20$ ) before adding MTT. a Between 1 and $24 \mathrm{~h}$ later, cell number was determined, and $\mathbf{b}$ cell viability was measured as described in "Materials and methods". Results are expressed as \% of the control $(100 \%)$. Values are the average of three separate experiments in quadruplicate and are expressed as mean \pm SD. ${ }^{*} p<0.05$.

unchanged in the presence of $5 \mu \mathrm{M}$ Rottlerin) are higher in the presence of $20 \mu \mathrm{M}$ Rottlerin due to its intrinsic absorbance at $340 \mathrm{~nm}$. Anyway, LDH activity and quantification are not altered by Rottlerin, the spectrophotometric interference of which does not invalidate LDH quantification. 


\section{Table 1}

\section{MTT reduction normalized to cell number}

\begin{tabular}{llc} 
& \multicolumn{2}{l}{ MTT/cell number } \\
\cline { 2 - 3 } Time (h) & R5 & R20 \\
\hline 1 & 1.23 & 1.54 \\
\hline 2 & 1.20 & 1.53 \\
\hline 4 & 1.20 & 1.70 \\
15 & 1.25 & 2.24 \\
\hline 24 & 1.58 & 3.49 \\
\hline
\end{tabular}

The MTT (\% control)/cell number (\% control) ratio was calculated in the samples treated with 5 and $20 \mu \mathrm{M}$ Rottlerin (R5 and R20, respectively) at the indicated time points

3.3. Rottlerin effect on the energy charge

3.4. Effect of mitochondrial uncoupling on MTT reduction
Because an immediate sign of mitochondrial uncoupling is the drop in cell phosphorylation potential, we evaluated the Rottlerin uncoupling effect by detecting the levels of all components of the adenylate pool using CZE (Fig. 4a) and then calculating the energy charge, represented by the ATP + ADP + AMP /0.5 (ATP + AMP). Rottlerin uncoupling effect is clearly evident at the dose of $20 \mu \mathrm{M}$, appearing as a fall in the cellular energy charge (Fig. 4b). The capacity to form high-energy phosphates was maximally compromised after $20 \mathrm{~min}(24 \%$ decrease $)$ and was recovered slowly thereafter. At $24 \mathrm{~h}$, cells recovered about $95 \%$ of the basal energy status, likely by compensatory metabolism. Rottlerin, at the dose of $5 \mu \mathrm{M}$, exerted a less evident effect (not statistically significant) on the cellular energy status, which was weakly compromised after $20-30$ min treatment (4-5\% decrease) and was completely recovered after $40 \mathrm{~min}$.

FCCP is a commonly used protonophore that collapses the mitochondria inner membrane potential disrupting mitochondria function (13). As shown in Fig. 5, a 30-min treatment of MCF-7 cells with 5 and $20 \mu \mathrm{M}$ FCCP caused a significant increase in MTT reduction, an effect similar to that achieved with Rottlerin at the same times and doses. This result suggests that the Rottlerin interference in the MTT assay is related to its ability to act as a mitochondrial uncoupler.

\section{Discussion}

The MTT assay is a widely used test to measure cell proliferation, cell viability/survival, or drug toxicity. In a recent paper, the use of 

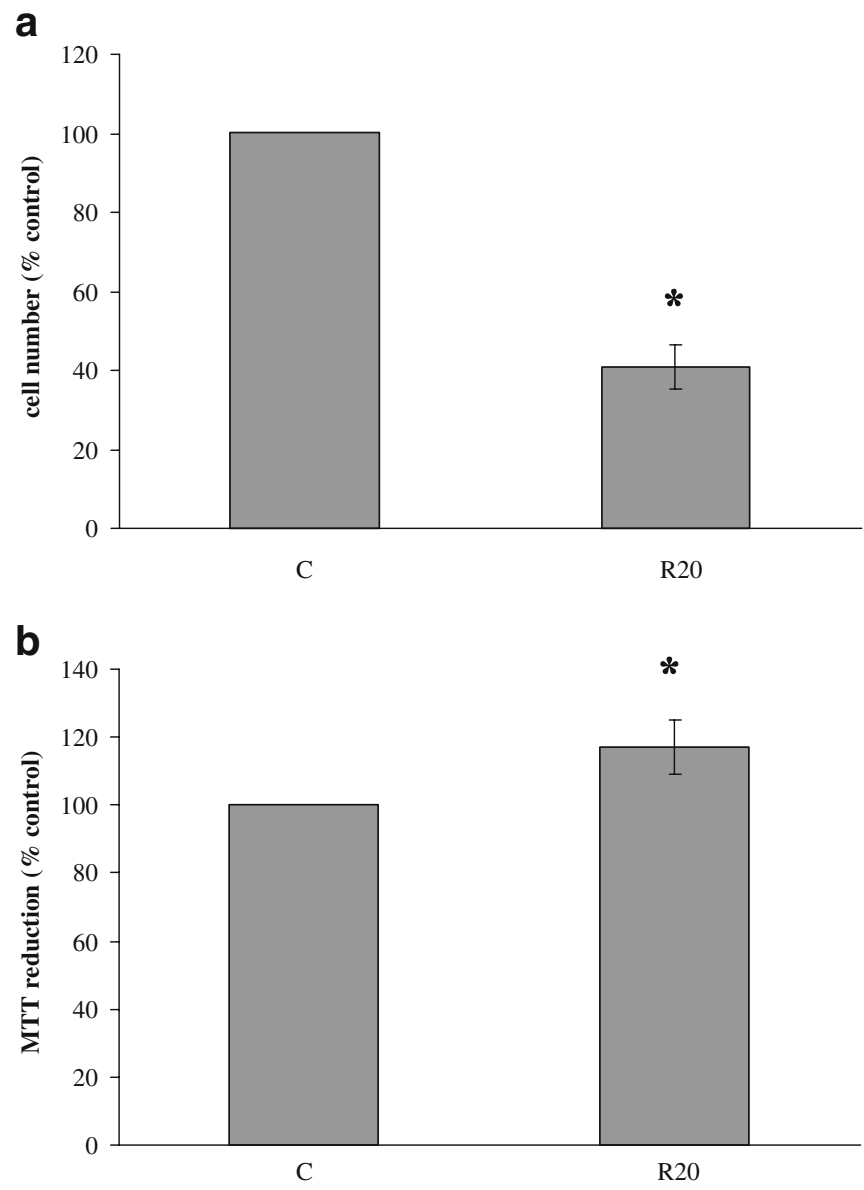

Fig. 3. Effect of Rottlerin on HMVEC cell number and viability assessed by direct cell counting and MTT assay, respectively. HMVEC were treated with $20 \mu \mathrm{M}$ Rottlerin (R20) before adding MTT. a Twenty-four hours later, cell number was determined, and $\mathbf{b}$ cell viability was measured as described in "Materials and methods". Results are expressed as \% of the control (100\%). Values are the average of three separate experiments in quadruplicate and are expressed as mean \pm SD. ${ }^{*} p<0.05$.

MTT has been described as one of the major techniques for testing tumor cell resistance to anticancer agents (14). On the other hand, several papers have reported agents that increase MTT reduction without increasing cell viability (15). Moreover, some studies reported that certain plant extracts and redox-active polyphenols can interfere with the MTT assay because they directly reduce the tetrazolium salt even in the absence of cells (16-19). Our results demonstrate that Rottlerin (5 and $20 \mu \mathrm{M}$ ) also strongly enhance the formation of formazan crystals inside cells. However, Rottlerin failed in reducing tetrazolium salts in vitro, indicating that the effect observed in cultured cells is not due to a direct reducing action but that the presence of an intermediate molecule/organelle is needed. 
Table 2

\section{LDH absorbance changes at $340 \mathrm{~nm}$ as a function of time in the presence or absence of Rottlerin}

DMSO

\begin{tabular}{llllll}
\cline { 2 - 3 } \cline { 5 - 5 } Time & OD & $\boldsymbol{\Delta A}$ & & OD & \multicolumn{1}{c}{$\boldsymbol{A}$} \\
\hline $30 \mathrm{~s}$ & 1.248 & & 1.365 & \\
\hline $1.5 \mathrm{~min}$ & 1.211 & 0.037 & 1.320 & 0.045 \\
\hline $2.5 \mathrm{~min}$ & 1.180 & 0.031 & 1.296 & 0.024 \\
\hline $3.5 \mathrm{~min}$ & 1.147 & 0.033 & 1.262 & 0.034 \\
\hline $4.5 \mathrm{~min}$ & 1.122 & 0.025 & 1.222 & 0.040 \\
\hline Mean $\Delta A / \mathrm{min}$ & 0.031 & & 0.035 & \\
\hline LDH $(\mathrm{U} / \mathrm{l})$ & 503 & & 568 & \\
\hline
\end{tabular}

The values reported in the table are representative of two $\mathrm{LDH}$ assays in the same sample and conditions

Because the MTT assay is largely based on mithocondrial LDH activity, we hypothesized that Rottlerin could enhance $\mathrm{LDH}$ activity indirectly, by maintaining high levels of NADH, due to its $\mathrm{H}+$ donor properties. Rottlerin indeed contains five phenolic hydroxyl groups (Fig. 1) that act as hydrogen donors in the scavenging of free radicals, such as 1,1-diphenyl-2-picrylhydrazyl (9). The results presented in the current study exclude any Rottlerin modulation of LDH activity in vitro, further confirming the need of whole cells to show the increase in MTT reduction.

Therefore, knowing that Rottlerin can interfere in the respiratory chain by acting as an uncoupler of oxidation and phosphorylation (4), we hypothesized that the Rottlerin artifact in the MTT test could be the consequence of a direct action on mitochondrial respiration. Rottlerin, indeed, by dissipating the inner mitochondrial membrane potential, accelerates electron transfer and increases dehydrogenases activity, oxygen consumption, and NADH oxidation. The observed drop in the cell energy charge indicated that also in MCF-7 cells Rottlerin exerted an uncoupling effect, which accidentally enhanced MTT reduction by over-stimulated mitochondrial dehydrogenases. To verify this hypothesis, a comparative study between Rottlerin and the chemical uncoupler FCCP was performed. The results indicated that the overestimation of the MTT test was linked to Rottlerin uncoupling properties, since both uncouplers, at the same doses, enhanced MTT reduction after only l-h incubation and roughly to the same extent.

The mitochondrial uncoupling was clearly evident with $20 \mu \mathrm{M}$ Rottlerin but only weakly apparent with $5 \mu \mathrm{M}$ Rottlerin. However, since this dose also caused overestimation of the 
a

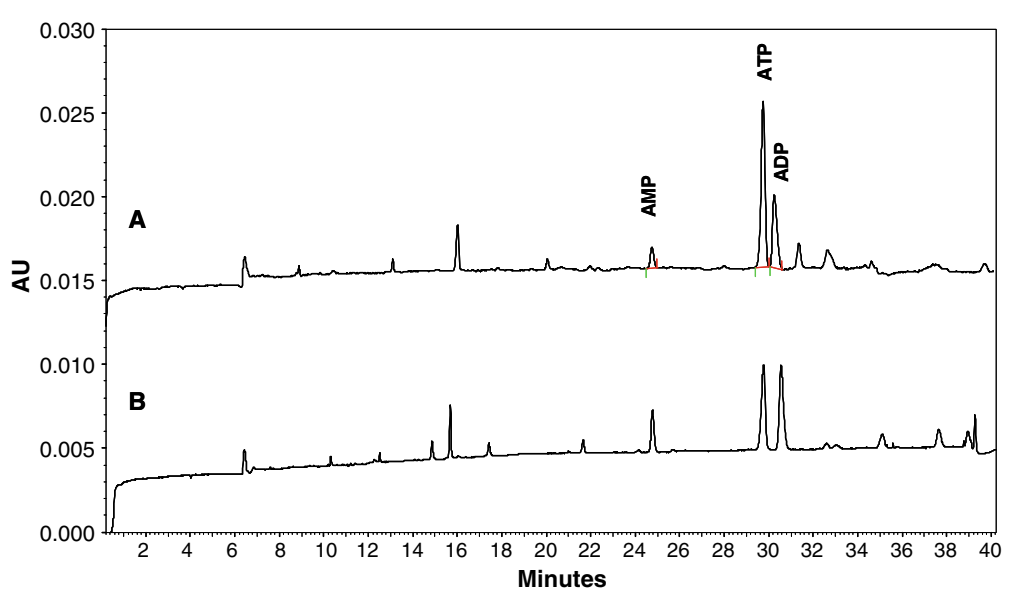

b

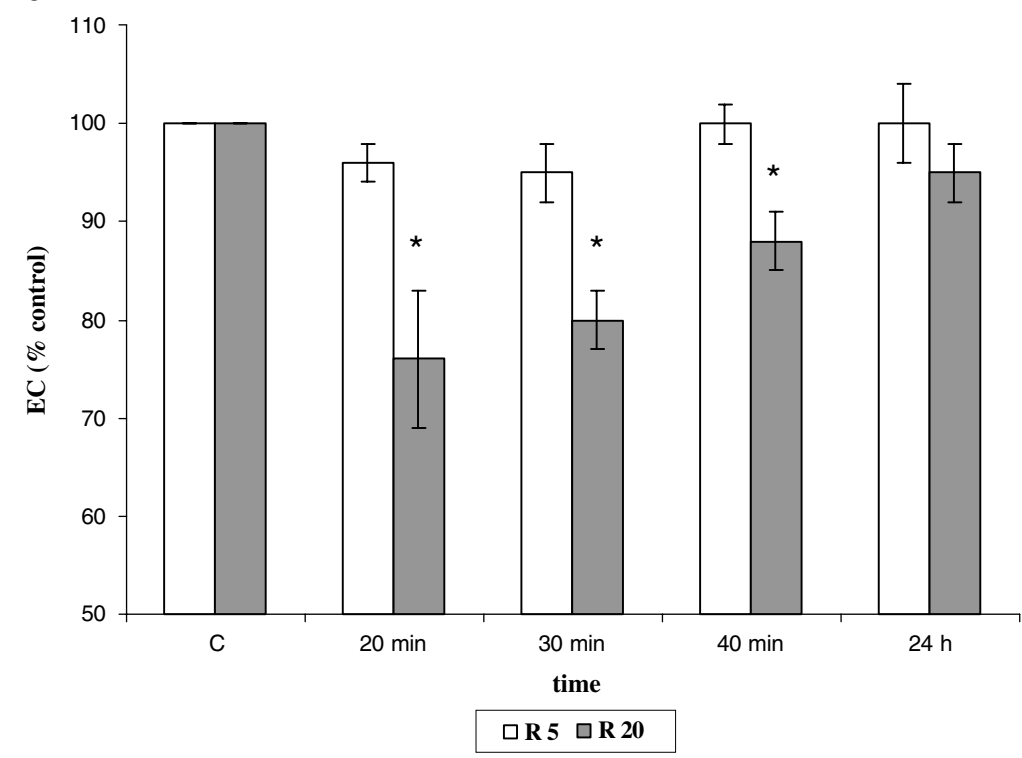

Fig. 4. Effect of Rottlerin on the energy charge (EO). Cells were treated with 5 and $20 \mu \mathrm{M}$ Rottlerin (R5and R2O), and high-energy phosphates (ATP, ADP, and $A M P$ ) were determined by CZE, as described in "Materials and methods". a Representative electropherogram of perchloric acid extracts from MCF-7 cells. A Control sample, B 30-min incubation with $20 \mu \mathrm{M}$ Rottlerin. $A U$ absorbance units. $\mathbf{b}$ The EC, represented by the ATP + ADP + AMP/0.5(ATP + AMP) ratio, was calculated at the indicated time points and expressed as $\%$ of the control (100\%). Values are the average of three separate experiments and are expressed as mean \pm SD. ${ }^{*} p<0.05$.

MTT test, it can be hypothesized that even with $5 \mu \mathrm{M}$ Rottlerin a mild uncoupling occurred in our cells, although could be promptly compensated, in terms of energy metabolism, by an increase in glycolytic ATP production. 


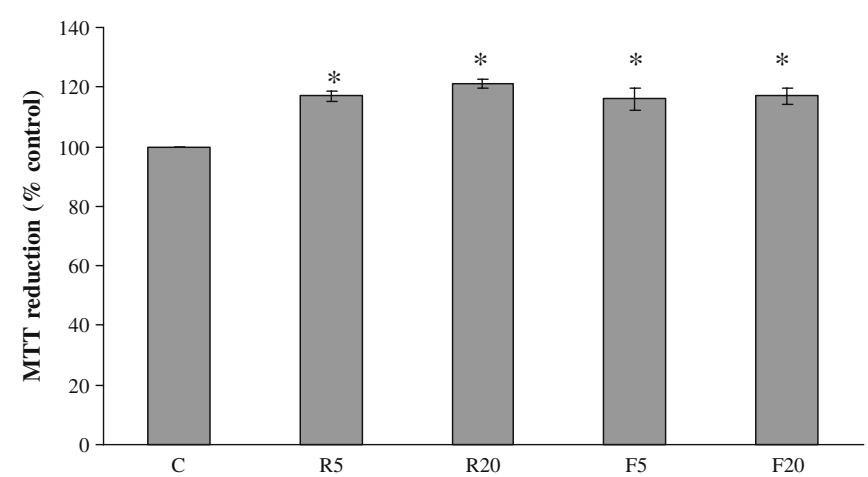

Fig. 5. Effect of Rottlerin and FCCP on MTT assay. Cells were treated for 30 min with 5 and $20 \mu \mathrm{M}$ Rottlerin and $\mathrm{FCCP}(R 5, R 20$ and $F 5$, F20, respectively) or vehicle alone (DMSO and ethanol, respectively) and MTT reduction was evaluated as described in "Materials and methods". Values are the average of three separate experiments in quadruplicate and are expressed as mean \pm SD. ${ }^{*} p<0.05$.

In the light of these new findings, we revised, in retrospect, our previous results about the lack of effect of Rottlerin on MCF-7 cell viability (5), since the overestimation of the MTT assay likely masked possible toxic/apoptotic effects in this cell line (false or underestimated result).

Considering that different cells may be not equally sensitive to uncouplers, we also tested the interference of Rottlerin in the MTT assay in another cell type. The results demonstrate that Rottlerin overestimates the MTT assay also in HMVEC, suggesting that such an artifact occurs independently from the cell type.

Our experience indicates that it may not be sufficient to change the medium containing Rottlerin and to wash the cells before adding MTT to avoid a potential bias in concluding results. In fact, the Rottlerin lipophylic nature allows the molecule to freely cross the plasma membrane, to accumulate inside the cell, to target mitochondria, and to exert the well documented uncoupling effects (4). The Rottlerin intracellular accumulation can be easily verified by a simple observation: The cells remain yellowcolored even after washing.

The results presented in the current paper also indicate that it may not be sufficient to include a control without cells in the MTT assay, a stratagem that has been recommended to avoid false-positive results in the presence of molecules with reducing properties, such as flavonoids (18). Rottlerin, indeed, does not react with MTT in vitro, but causes enhanced MTT reduction in cultured cells.

Similar to the MTT, the 2,3-bis-(2-methoxy-4-nitro-5sulfophenyl)-2H-tetrazolium-5-carboxanilide (XTT) measures cell viability based on the activity of mitochondria enzymes in live cells. Unlike the water-insoluble formazan produced from MTT, XTT is readily reduced to a highly water-soluble orange colored 
product, thus eliminating the need for the solubilization step required for the MTT assay.

Similar to the XTT, the Dojindo's tetrazolium salt, the 2-(2methoxy-4-nitrophenyl)-3-(4-nitrophenyl)-5-(2,4-disulfophenyl)$2 \mathrm{H}$ tetrazolium, monosodium salt (WST-8), marketed as Cell Counting kit-8 (CCK-8), is reduced by cellular dehydrogenases, in the presence of an electron carrier, to an orange formazan product that is water-soluble.

Despite the technical differences and the detection sensitivity, the MTT, XTT, and WST-8 work on exactly the same principle and measure the same parameter. Therefore, it can be anticipated that the uncoupling properties of Rottlerin also could have a detrimental effect on the results from both XTT and WST-8 cell proliferation assays.

On the basis of our findings, it can be suggested that a safety alternative test in toxicological studies could be the detection of $\mathrm{LDH}$ released in the medium by cultured cells, since no interference was observed in our LDH assay in the presence of Rottlerin in the most widely used concentration range $(5-20 \mu \mathrm{M})$. Some other $\mathrm{LDH}$ commercial kits are based on a two steps reaction: (1) the oxidation of lactate to pyruvate and (2) reduction of MTT to formazan by pyruvate. Also these $\mathrm{LDH}$ assays can be recommended in the presence of Rottlerin, the drug did not exhibit any direct reactivity toward MTT salts in vitro; thus no interference should be expected.

Moreover, we also discourage the use of the MTT assay in the presence of mitochondrial uncoupling agents; a number of lipophylic molecules, both natural and synthetic, such as polyphenols and steroid hormones, have uncoupling properties. In these cases, the direct cell counting or the direct measurement of cell proliferation (tritiated thymidine or others) seems to be more appropriate than the MTT assay.

Another widely used method to assess proliferation, cytotoxicity, or viability of cultured mammalian cells and to monitor the effects of a wide range of drugs and biological compounds is the measurement of ATP content in lysed cells by colorimetric, fluorometric, and radioisotopic assays. ATP is a good marker for cell viability because it is present in all metabolically active cells, and the concentration declines very rapidly when the cells undergo necrosis or apoptosis. However, although we have not verified a possible interference by Rottlerin and other uncouplers in ATP detection by the methods above, it is possible, or better obvious, that the decrease in ATP caused by mitochondrial uncoupling could be erroneously interpreted as a decrease in cell viability (false or enhanced negative result). We have no suggestions on how to circumvent the interference in these cases and can only discourage the use of ATP measurement in the presence of known or putative uncoupling molecules. 
In closing, we have demonstrated in this report that, although the MTT method is the most frequently used in the assessment of the viable cell number, its inadequacy is evident in presence of drugs/compound that cause alterations in mitochondria activity. We observed a significant pitfall in the presence of the chemicals tested, resulting in inaccurate cell number estimation and misinterpretation of results. Therefore, we suggest caution in the use of the MTT assay as a proliferation/viability/toxicity test in the presence of Rottlerin and uncoupling agents in general, especially if not corroborated using alternative/complementary assays.

\section{Acknowledgment}

\section{References}

1. Gschwendt M, Mülle HJ, Kielbassa K, Zang R, Kittstein W, Rincke G et al (1994) Rottlerin, a novel protein kinase inhibitor. Biochem Biophys Res Commun 199(1):93-98

2. Davies SP, Reddy H, Caivano M, Cohen P (2000) Specificity and mechanism of action of some commonly used protein kinase inhibitors. Biochem J 35l(Pt 1):95-105

3. Soltoff SP (2007) Rottlerin: an inappropriate and ineffective inhibitor of PKCdelta. Trends Pharmacol Sci 28(9):453-458

4. Soltoff SP (2001) Rottlerin is a mitochondrial uncoupler that decreases cellular ATP levels and indirectly blocks protein kinase Cdelta tyrosine phosphorylation. J Biol Chem 276(41):37986-37992

5. Torricelli C, Fortino V, Capurro E, Valacchi G, Pacini A, Muscettola M et al (2008) Rottlerin inhibits the nuclear factor $\mathrm{kappaB} /$ cyclin-Dl cascade in MCF-7 breast cancer cells. Life Sci 82(11-12):638-643

6. Valacchi G, Pecorelli A, Mencarelli M, Carbotti P, Fortino V, Muscettola M et al (2009) Rottlerin: a multifaced regulator of keratinocyte cell cycle. Exp Dermatol 18(6):516-521

7. Kontny E, Kurowska M, Szczepańska K, Maśliński W (2000) Rottlerin, a PKC isozyme-selective inhibitor, affects signaling events and cytokine production in human monocytes. J Leukoc Biol 67(2):249-258

8. Storz P, Döppler H, Toker A (2004) Protein kinase Cdelta selectively regulates protein kinase $\mathrm{D}$-dependent activation of
NF-kappaB in oxidative stress signaling. Mol Cell Biol 24(7):2614-2626

9. Longpre JM, Loo G (2008) Protection of human colon epithelial cells against deoxycholate by Rottlerin. Apoptosis 13(9): 1162-1171

10. Banno T, Gazel A, Blumenberg M (2005) Pathway-specific profiling identifies the NF-kappa B-dependent tumor necrosis factor alpha-regulated genes in epidermal keratinocytes. J Biol Chem 280(19): 18973-18980

11. Mosmann T (1983) Rapid colorimetric assay for cellular growth and survival: application to proliferation and cytotoxicity assays. J Immunol Methods 65(1-2):55-63

12. Carlucci F, Tabucchi A, Biagioli B, Sani G, Lisi G, Maccherini M et al (2000) Capillary electrophoresis in the evaluation of ischemic injury: simultaneous determination of purine compounds and glutathione. Electrophoresis 21(8):1552-1557

13. Terada H (1981) The interaction of highly active uncouplers with mitochondria. Biochim Biophys Acta 639(3-4):225-242

14. Hatok J, Babusikova E, Matakova T, Mistuna D, Dobrota D, Racay P (2009) In vitro assays for the evaluation of drug resistance in tumor cells. Clin Exp Med 9 (1):1-7

15. Sims JT, Plattner R (2009) MTT assays cannot be utilized to study the effects of STI571/Gleevec on the viability of solid tumor cell lines. Cancer Chemother Pharmacol 64(3):629-633 
16. Bruggisser $\mathrm{R}$, von Daeniken $\mathrm{K}$, Jundt $\mathrm{G}$, Schaffner W, Tullberg-Reinert H (2002) Interference of plant extracts, phytoestrogens and antioxidants with the MTT tetrazolium assay. Planta Med 68(5):445-448

17. Shoemaker M, Cohen I, Campbell M (2004) Reduction of MTT by aqueous herbal extracts in the absence of cells. J Ethnopharmacol 93(2-3):381-388
18. Peng L, Wang B, Ren P (2005) Reduction of MTT by flavonoids in the absence of cells. Colloids Surf B Biointerfaces 45 (2):108-111

19. Wisman KN, Perkins AA, Jeffers MD, Hagerman AE (2008) Accurate assessment of the bioactivities of redox-active polyphenolics in cell culture. J Agric Food Chem 56(17):7831-7837 